\title{
Physical and Chemical Properties of GdN: A Critical Comparison between Single Crystals and Thin Films, Theory and Experiment
}

\author{
Peter Wachter \\ Laboratorium für Festkörperphysik, ETH Zürich, Zürich, Switzerland \\ Email:wachter@solid.phys.ethz.ch
}

Received 14 January 2016; accepted 11 March 2016; published 14 March 2016

Copyright (c) 2016 by author and Scientific Research Publishing Inc.

This work is licensed under the Creative Commons Attribution International License (CC BY).

http://creativecommons.org/licenses/by/4.0/

(c) (i) Open Access

\section{Abstract}

Since about 10 years, there is a controversy about physics and chemistry of GdN between stoichiometric (tested) large single crystals and off-stoichiometric thin films. GdN single crystals are antiferromagnetic for applied magnetic fields of only $10 \mathrm{0e}$, become ferromagnetic for excess electrons and larger magnetic fields. They are semimetallic. Thin films are ferromagnetic and semiconductors. Over the time, many experiments have been performed on both systems and the physics in each system is consistent. Band structure computations either yield ferromagnetic semimetals or ferromagnetic semiconducting thin films. There seems to be two incompatible worlds, those of single crystals and those of thin films. In the present work, the author compares directly the various measurements and calculations and gives reasons for their different results.

\section{Keywords}

Rare Earth Nitrides, Preparation Methods, Magnetic, Electrical and Optical Measurements on Single Crystals and Thin Films

\section{Introduction}

The history of rare earth mono-nitrides is a long lasting (more than 50 years) story of errors, misunderstanding, limited measuring techniques and undefined samples. Fundamental questions arise due to the conducting behavior, semiconductors, semimetals or metals and to the magnetic behavior, ferro-, ferri-, antiferro- or metamagnets [1] [2].

First magneto-chemical measurements have been performed in the late 50ies on poly-crystals of undefined composition. The problem rests in the fact that nitrogen is a gas whereas for the other rare earth pnictides the 
pnictogen is a solid. The latter enables easy mixing of the constituents and at high enough temperatures the chemical reaction starts, but the stoichiometry of the resulting crystals has rarely been checked. Handbook articles about rare earth pnictides are in Ref. [1] [2]. Stoichiometry (from Greek $\sigma \tau o \imath \chi \varepsilon \imath o v \mu \varepsilon \tau \rho \varepsilon \imath v$ basic material measure) is the key element for all rare earth pnictides, but especially for the nitrides. Exact stoichiometry means e.g. $\mathrm{Gd}_{1.0} \mathrm{~N}_{1.0}$, but all pnictides generally have metal excess up to $10 \%$, e.g. $\mathrm{Gd}_{1.0} \mathrm{~N}_{0.9}$ or $\mathrm{Gd}_{1.1} \mathrm{~N}_{1.0}$ [1] [3] As will be more elaborated later on, each excess metal ion will donate excess electrons and turn a possible semiconductor into a semimetal or metal. The magnetic interactions are at least composed of two parts-an indirect superexchange involving 4f-bonding and antibonding with d-orbits of the cation and the p orbits of the anionand a RKKY type interaction where the oscillatory behavior depends on the carrier concentration. Thus a stoichiometric nitride may be an antiferromagnet, but becomes a ferromagnet with off-stoichiometry. In this paper we use the term stoichiometry in short for a 1:1 exact stoichiometry, else wise we use the term off-stoichiometry [3].

Why is exact stoichiometry so important for rare earth mono-nitrides? The free carrier concentration as measured with the Hall effect is between $10^{19} \mathrm{~cm}^{-3}$ and $10^{21} \mathrm{~cm}^{-3}$ for various rare earth nitride single crystals [3], thus only percents of the cation concentration. If one is off-stoichiometric in the percentage range, the carrier concentration may change by more than $100 \%$ and thus change all physical and chemical characters. This is unusual when compared with other "normal” compounds, where a few percent off-stoichiometry change physical and chemical parameters also only by percents and may be tolerated.

However, the degree of stoichiometry must be measured experimentally and not only claimed. Very often it is the crystal structure and rocksalt phase purity, which is used as a sign of stoichiometry. Even in a recent investigation on rare earth nitrides [4] the phase purity from XRD has been used to claim perfect stoichiometric polycrystalline samples. But it is a fact that non-stoichiometry up to $10 \%$ is still single phase with only the rocksalt structure. Of course, there is a large diffuse background since the lattice defects are disordered and do not exhibit another crystallographic phase. But when one insists on using 99.99\% pure rare earths and 99.9995\% pure N and thus hoping to get perfect samples, one has not understood the problems with non-stoichiometry [4]. Some authors realize or even measure the off-stoichiometry of their samples but think this would not change the physics of the material. But it does, as will be shown further down. So Child et al. [5] measured with the Kjeldahl method about 95\% nitrogen and Sclar [6] about 90\% [7].

All rare earth nitride stoichiometric crystals are metallic conducting (the other pnictides just as well) when measured in function of temperature [7]. The point is: are they metallic because of excess metal electrons due to non-stoichiometry or is this an intrinsic property due to the band structure and p-d overlap? In any case those nitrides, which have the lowest carrier concentration and perfect measured stoichiometry are the best and serve as intrinsic standards.

In the last decade, GdN films have been introduced with drastically different physical properties compared with stoichiometric single crystals. They seem to be ferromagnetic and semiconducting, whereas stoichiometric single crystals are (for very low magnetic fields) antiferromagnets and their conducting behavior is metallic (semimetallic). We want to compare in this paper the properties of single crystals and thin films and give a hint why thin films are so different. Part of the Introduction and Crystal Growth stem from an earlier paper [7].

\section{Preparation of GdN Single Crystals}

A short review of various preparation methods for polycrystalline $\mathrm{GdN}$ and various physical measurements on it are given in Ref. [3].

Whereas the former all yield polycrystalline powders of various compositions, single crystals of the nitrides can also be grown [8] [9]. In short, the handling of the metal and the compound has been performed in metallic argon glove boxes gettered by hot cerium turnings, having a residual oxygen concentration of less than 3 ppm. Commercially available 99.99\% sublimed rare earth metal has been transformed into metal turnings in the glove box and pre-reacted with an induction furnace in an open Mo crucible for $12 \mathrm{~h}$ at $1900^{\circ} \mathrm{C}$ in a nitrogen stream. The resulting polycrystalline material has been reground in the glove box and nitrided again for $12 \mathrm{~h}$ and the procedure repeated again. Even after 24 h of X-ray irradiation only the rocksalt structure could be detected and the lattice constant was $4.984 \AA$ A. Single crystals have been analyzed with a micro Kjeldahl method [9] for their nitrogen content and were found to be stoichiometric $( \pm 0.5 \%)$. The Kjeldahl method is a wet chemical analysis, where finally a titration determines the nitrogen content. The large single crystals permitted the method to be applied on even one crystal [3]. 
In short: $\mathrm{GdN}+\mathrm{H}_{2} \mathrm{SO}_{4} \rightarrow\left(\mathrm{NH}_{4}\right)_{2} \mathrm{SO}_{4}+$ others.

$\left(\mathrm{NH}_{4}\right)_{2} \mathrm{SO}_{4}+2 \mathrm{NaOH} \rightarrow \mathrm{Na}_{2} \mathrm{SO}_{4}+2 \mathrm{NH}_{3}+2 \mathrm{H}_{2} \mathrm{O}$.

$\mathrm{NH}_{3}+$ acid $\rightarrow$ titration.

Precision for nitrogen: $\pm 0.5 \%$.

As is well known a titration is the most precise chemical analysis.

This prereacted material has been put into a tungsten crucible closed and welded with an electron beam. A temperature gradient of $1940^{\circ} \mathrm{C}$ and $1970^{\circ} \mathrm{C}$ between top and bottom of the crucible has been maintained for 11 days and then the crucible has been slowly cooled. Large single crystals between $3 \mathrm{~mm}$ and $5 \mathrm{~mm}$ edge length had grown. Within the precision of the chemical analysis no nitrogen has been lost. Single crystals (with Laue pattern) from this crucible have been cleaved in the glove box and been transferred without contamination with air into the various measuring apparatuses. Single crystals from top or bottom of the crucibles, though with the same stoichiometry, could have slightly different physical properties. So all physical measurements should be done on the very same single crystal. In Figure 1 we show one of the tungsten crucibles and a collection of GdN single crystals [3] [7].

The perfection and quality of GdN is extremely important. A change in stoichiometry of only $1 \%$ may change the carrier concentration by $100 \%$ and thus influence all physical parameters like lattice constant, magnetic and optical properties and electric conductivity.

A simple test of the perfection of the nitride material is to leave a polycrystalline sample over night on the table in the laboratory. On the next morning an originally non-stoichiometric black powder has become a white material, e.g. $\mathrm{Gd}_{2} \mathrm{O}_{3}$ or $\mathrm{Gd}(\mathrm{OH})_{3}$. Instead a stoichiometric single crystal is stable in air and could be boiled for hours in a mixture of $\mathrm{HNO}_{3}$ and $\mathrm{H}_{2} \mathrm{SO}_{4}$ before being dissolved.

In this paper we want to compare and measure physical properties of stoichiometry tested (Kjeldahl), large single crystals of GdN and relate them to thin film data of unknown stoichiometry. The results are drastically different. Then we want to test theoretical band structure calculations amongst each other and relate them to various experiments.

\section{Preparation of Thin Films of GdN}

Various methods to grow thin films of GdN have been used in the past. Thin films always need substrates to be grown on, sometimes Si (100) [10], sometimes sapphire in the c-direction [11]. Generally, the GdN films have been covered with a layer to protect them against oxidation, mostly GaN or $\mathrm{MgF}_{2}$ (if they would have been stoichiometric they would be stable in air). In preparing thin films of GdN sometimes reactive sputtering of nitrogen atoms on a growing Gd film was used, hoping to get only GdN molecules [10] or thin films where grown by thermal co-evaporation of $\mathrm{Gd}$ in a $\mathrm{N}_{2}$ atmosphere on supports at room temperature [11]-[16]. Always the GdN films have been capped with $\mathrm{GaN}$ or $\mathrm{MgF}_{2}$. Sometimes nano-crystallinity has been observed [13], some-

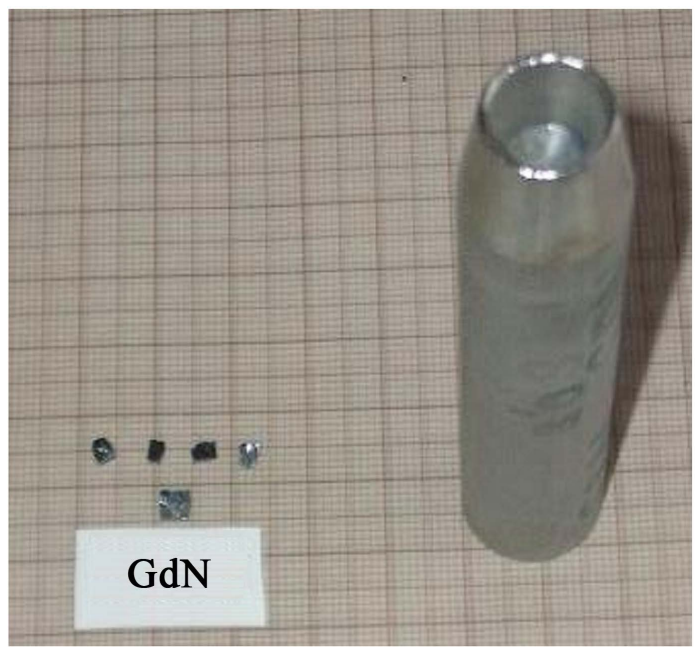

Figure 1. Tungsten crucible (about $10 \mathrm{~cm}$ in height) and GdN single crystals. 
times epitaxy [12]. The reported lattice constants of GdN films were $5.05 \AA$ [10], $5.12 \AA$ [14], $5.01 \AA$ [13], 5.00 $\AA$ [12], $5.04 \AA$ [11] [15]. They are appreciable larger than the 4.98X $\AA$ accepted for polycrystalline powder samples since about 50 years [17] and $4.984 \AA$ for GdN stoichiometric single crystals [3] [7] [18]. The reason must be that there are large voids in the films, which can be occupied by $\mathrm{N}_{2}$ molecules or $\mathrm{N}$ ions, which absorb surplus charge carriers, making the films semiconducting. However, when using sapphire as support, crystalline hexagonal with lattice constant in the c-direction of $12.9 \AA$ and a GaN cover with wurtzit lattice of $5.185 \AA$ [11] [13]-[15] it is clear that the GdN film is stretched in the plain. This would make the crystal structure tetragonal. However, all authors claim cubic symmetry, but nobody gives a proof for that. As will be shown further down in theory, stretching GdN can make it semiconducting, high pressure experiments on single crystals make them more metallic (unpublished results by the author).

In Figure 2 we show in a 2-dimensional model of equal spheres that one must have always voids in a 2-dimensional lattice, independent how often you shake it. The voids change, but are always there. In these voids additional nitrogen ions or molecules can be stored, stretching the lattice, but absorbing also charge carriers.

In other words, thin films of GdN as prepared with larger lattice constants than stoichiometric single crystals, do not represent GdN in its ground state. The stoichiometry of these films has never been measured and cannot be measured with the necessary precision of less than $1 \%$, some of the authors even state that their films are non-stoichiometric [10] [15].

The decades long experience of the author in preparing thin films of rare earth chalcogenides with methods described above failed to give the same results as obtained on single crystals. Thus, what we called a "flash evaporation" finally gave comparable results with single crystals and is recommended also for rare earth pnictides. In the evaporation chamber a white glowing current heated tungsten sheet was mounted and a contraption built to drop (if possible) stoichiometric polycrystalline powder from above on the glowing tungsten sheet. A big cloud of e.g. GdN molecules condensed everywhere and also on a substrate with various temperature. It is a big difference to condense GdN molecules on a substrate rather than try to nitride condensed Gd films.

\section{Magnetic Measurements}

Magnetic measurements are the core information on rare earth pnictides [7], especially on GdN [3] [7] [18] and they have been performed from the first investigations on pnictides for the last 50 or so years. An epic discussion has been whether GdN is ferromagnetic, antiferromagnetic or metamagnetic. In the past the magnetic measurements on polycrystalline material of unknown stoichiometry have been made (also in this institution) with applied magnetic fields in the order above $10 \mathrm{kOe}$, and no doubt, the material is ferromagnetic with a $\mathrm{T}_{\mathrm{c}}$ near 70 $\mathrm{K}$ and a saturation magnetization of $7 \mu_{\mathrm{B}}$ per Gd ion (references in part in [7]). However, such a strong magnetic field is a severe magnetic excitation and does not represent the magnetic ground state of GdN. The magnetic properties are defined for $\mathrm{H} \rightarrow 0$ and with modern methods small magnetic fields between 5 and 10 Oe can be

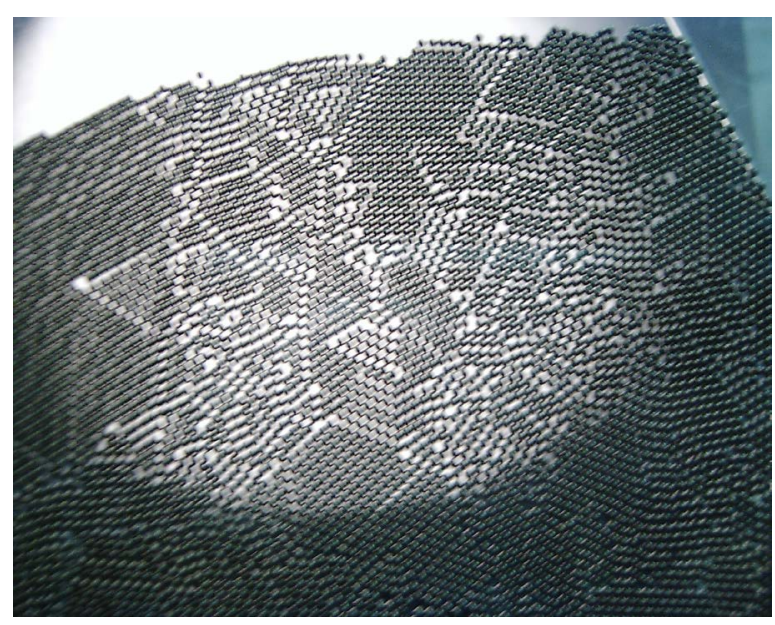

Figure 2. 2-dimensional model of equal metallic spheres $(0.5 \mathrm{~mm}$ diameter) between two plexiglass walls. The white dots are the voids, where balls are missing. (Mandelbrot pattern). 
applied for films, polycrystals and single crystals. This has been done already 1972 and as a first time stoichiometric GdN in a field of 10 Oe was shown to be antiferromagnetic with a $T_{N}$ around $25 \mathrm{~K}$ [17]. In Figure 3, we reproduce the original figure. In the upper part are GdN polycrystalline probes of unknown stoichiometry but with excess Gd and with various preparation methods in a magnetic field of $13 \mathrm{kOe}$. They clearly have a ferromagnetic signature. But there are also sublimed small (several tenths of $\mathrm{mm}$ ) single crystals which have been tested for stoichiometry with the Kjeldahl method, not on one single crystal, but for an average of small crystals and they have been found stoichiometric. In the lower part of the figure are magnetic measurements in low fields of only 10 Oe. Clearly these sublimed stoichiometric crystals are antiferromagnetic. The curve without markings has been added from measurements on a single large stoichiometric crystal [18] and was also antiferromagnetic, but with a larger $T_{N}$ of $40 \mathrm{~K}$ than the sublimed crystals [17]. It is obvious, that fine details in the stoichiometry are responsible for a small variation of $\mathrm{T}_{\mathrm{N}}$.

In Figure 4 it can be seen that only $\mathrm{GdN}_{0.96} \mathrm{O}_{0.04}$ with excess electrons is ferromagnetic from the beginning, whereas stoichiometric GdN single crystals start with a smaller inclination for low fields, again indicating antiferromagnetism. The saturation magnetism is $7 \mu_{\mathrm{B}}$ for both crystals.

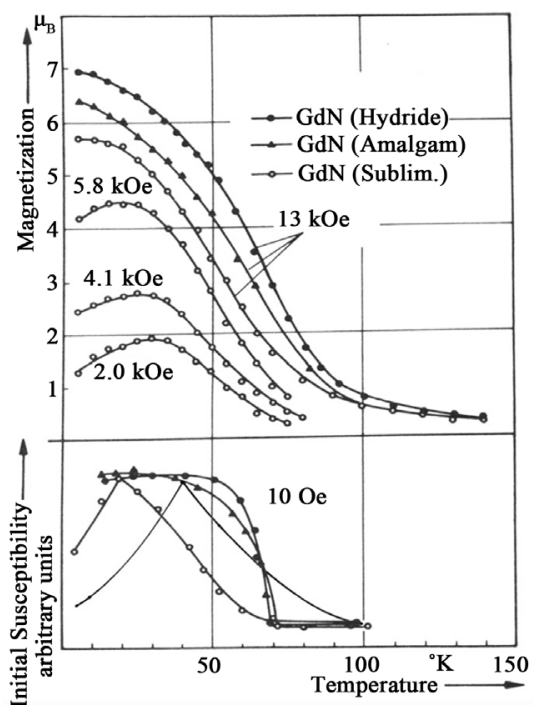

Figure 3. Magnetisation and initial susceptibility of GdN [17].

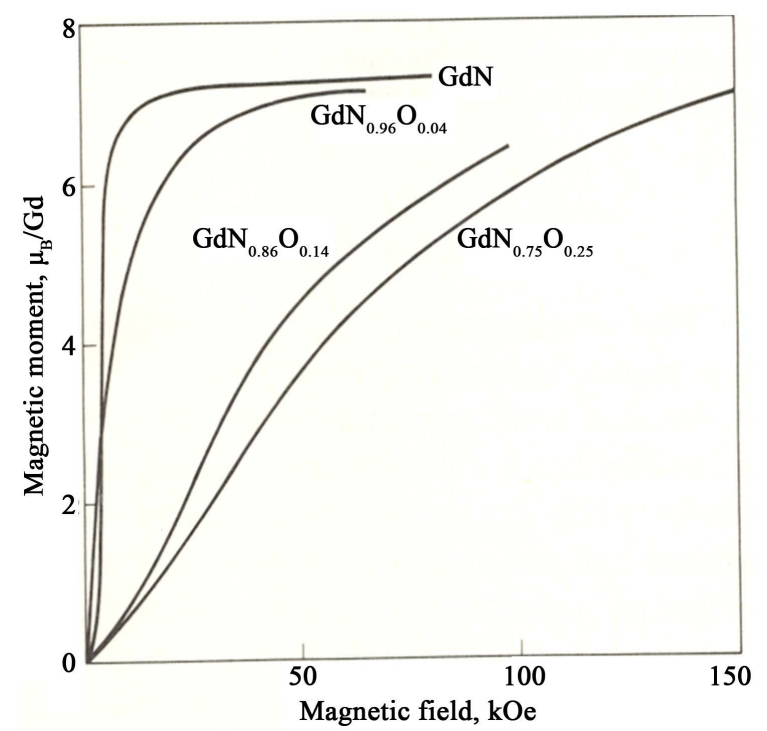

Figure 4. Magnetisation of GdN and $\mathrm{GdN}_{1-\mathrm{x}} \mathrm{O}_{\mathrm{x}}$ single crystals [18]. 
To rule out all questions about the magnetic behavior of stoichiometric GdN single crystals we have measured these crystals with a SQUID magnetometer [3] [7]. The sensitivity of this magnetometer was so large that the crystal had to be cleaved so small that a directional orientation was not possible anymore. In Figure 5(a) and Figure 5(b) we show the magnetic susceptibility and the inverse susceptibility, both in a field of only 10 Oe, and clearly the signature is antiferromagnetic, with a $\mathrm{T}_{\mathrm{N}}$ of about $33 \mathrm{~K}$.

In Figure 6 we show the magnetisation and inverse susceptibility of poly-crystalline GdN of unknown stoichiometry in a large magnetic field of $13 \mathrm{kOe}$. Clearly it is ferromagnetic with a $\mathrm{T}_{\mathrm{C}}$ of about $70 \mathrm{~K}$ as it has been measured for the last 50 years. But comparing the inverse susceptibility of Figure 6 with the one in Figure 5(b) (in these measurements domains do not play a role) one realizes a great difference. Since Figure 6 represents ferromagnetism, this cannot be the case in Figure 5(a) and Figure 5(b), it must be antiferromagnetism. So we learn, for small magnetic fields of 10 Oe, representing $\mathrm{H} \rightarrow 0$, stoichiometric GdN single crystals are antiferromagnetic with $\mathrm{T}_{\mathrm{N}}$ at about $33 \mathrm{~K}$, in larger fields they become ferromagnetic with a $\mathrm{T}_{\mathrm{C}}$ of about $70 \mathrm{~K}$. This behavior is called metamagnetism. Non-stoichiometric GdN crystals or stretched GdN films can be ferromagnetic, but low field measurements on films have rarely been performed. In Figure 7 the RKKY contribution alone to $\mathrm{GdN}$ is represented and will be discussed further down.

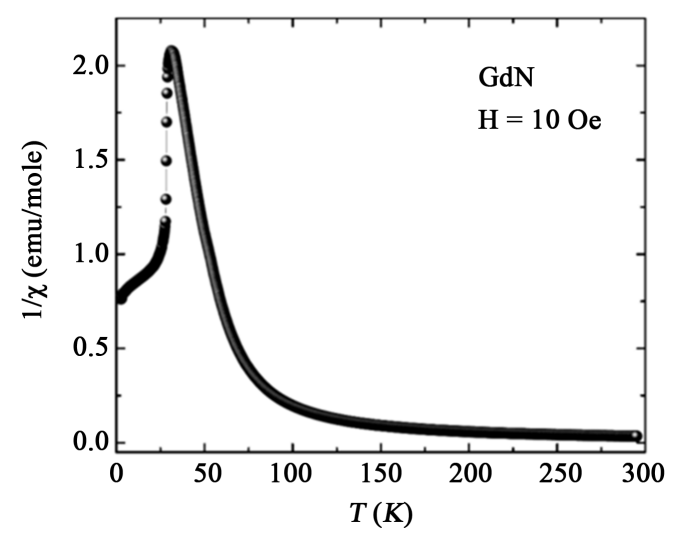

(a)

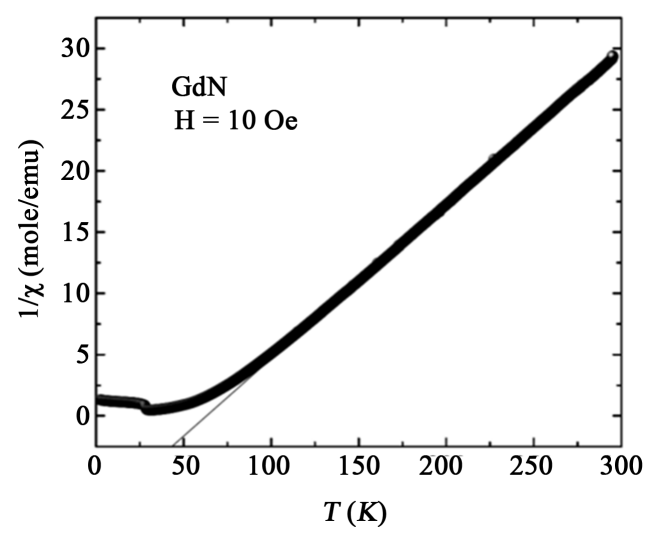

(b)

Figure 5. (a) The susceptibility of a small GdN single crystal in a field of 10 Oe [3] [7]; (b) The inverse magnetic susceptibility of a small GdN single crystal in a field of 10 Oe [3] [7].

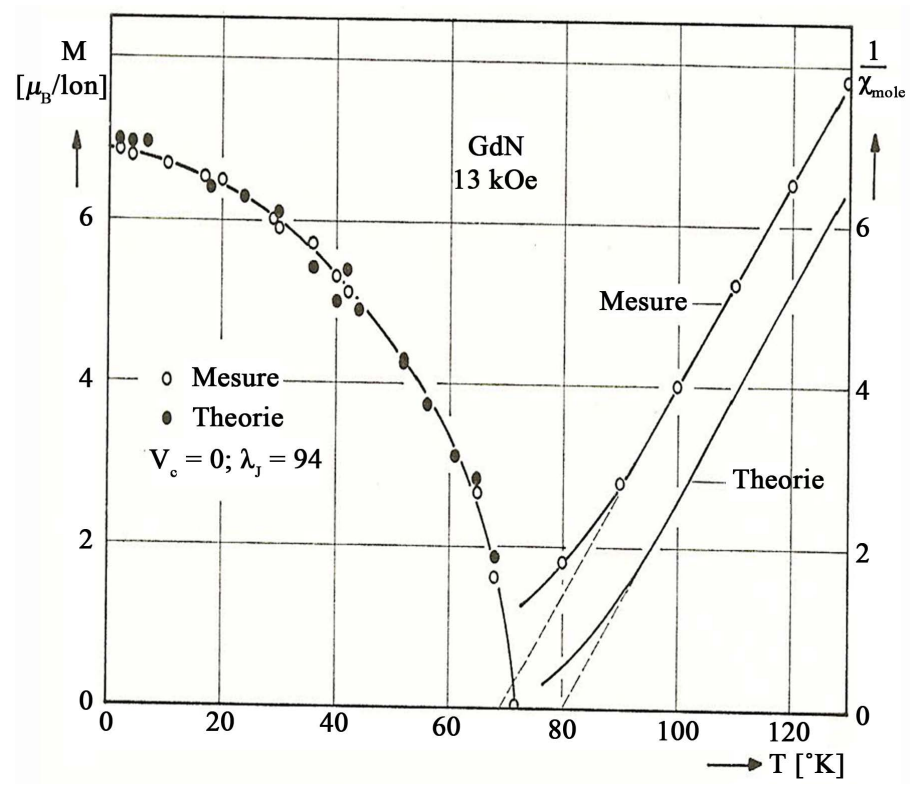

Figure 6. Magnetisation and inverse susceptibility of GdN [26]. 


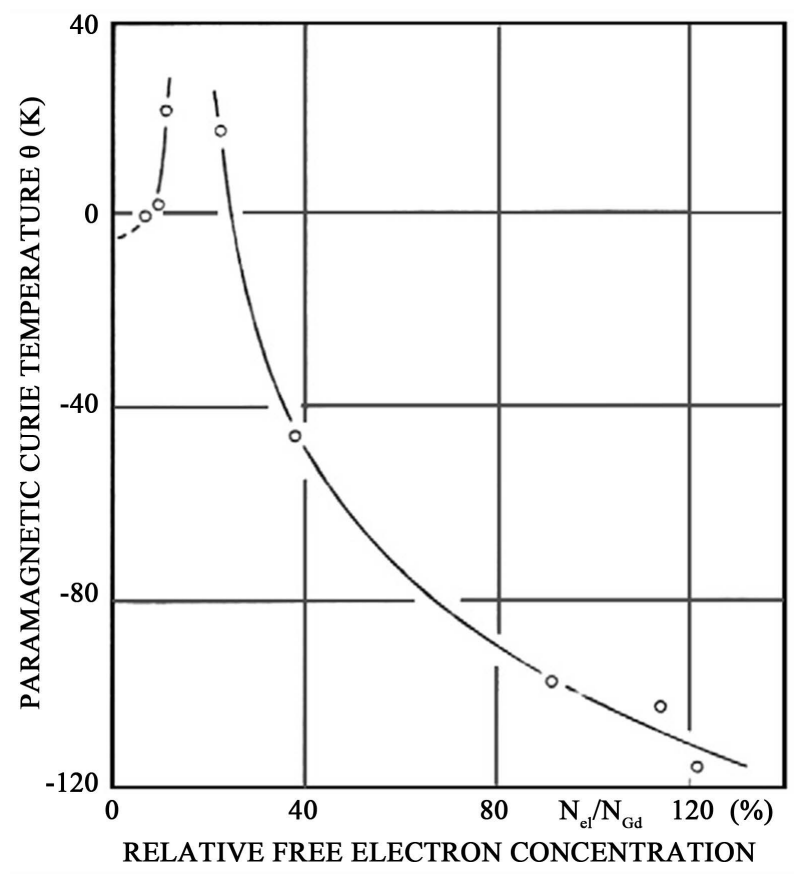

Figure 7. Paramagnetic Curie temperature versus relative free electron concentration of GdP, GdP/GdS and GdS [27].

\section{Magnetic Exchange Interactions}

To evaluate the magnetic exchange interactions it is essential to know the position of the $4 \mathrm{f}^{7}$ localized levels below the Fermi level. In Ref. [16] a comparison has been made of GdN with EuO inasmuch as divalent Eu and trivalent Gd have the same $4 \mathrm{f}^{7}$ level, the same crystal structure and both are assumed to be ferromagnetic (but see above). But there are distinct differences: divalent Eu has a much larger ionic diameter than trivalent Gd. As a consequence the lattice constant in EuO is thus $5.141 \AA$ compared with $4.984 \AA$ in GdN single crystals. Thus the $5 \mathrm{~d}$ and $2 \mathrm{p}$ derived bands are wider in $\mathrm{GdN}$, the crystal field splitting of the $5 \mathrm{~d}$ band in $\mathrm{dt}_{2 \mathrm{~g}}$ and $\mathrm{de}_{\mathrm{g}}$ is larger and the sequence of bands in EuO $\left(2 \mathrm{p}^{6}-4 \mathrm{f}^{7}-5 \mathrm{dt}_{2 \mathrm{~g}}\right)$ is different in GdN $\left(4 \mathrm{f}^{7}-2 \mathrm{p}^{6}-5 \mathrm{dt}_{2 \mathrm{~g}}\right)$. A calculation using tabulated ionization potentials has been done already 1978 [19] and a comparison beween EuS and GdN has been made and in 1979 [20] a review paper about the Eu chalcogenides has been published. One does not need band structure calculations to realize this. The most important difference between both compounds is the position of the $4 \mathrm{f}^{7}$ level below the Fermi level. In this calculation with ionization potentials using a Haber-Born thermo-chemical process the $4 \mathrm{f}^{7}$ state in EuS is found about $1 \mathrm{eV}$ below the Fermi level (in the gap between $4 \mathrm{f}^{7}$ and $5 \mathrm{dt}_{2 \mathrm{~g}}$ ) and in $\mathrm{GdN}$ it is $9 \mathrm{eV}$ below the Fermi level, which is cutting $2 \mathrm{p}^{6}$ and $5 \mathrm{dt}_{2 \mathrm{~g}}$. XPS photoemission is the right experimental method to measure the position of the $4 \mathrm{f}$ levels in rare earths. In photoemission always the final state is shown, in XPS from a $4 \mathrm{f}^{7}$ level this is the $4 \mathrm{f}^{6}{ }^{7} \mathrm{~F}_{\mathrm{J}}$ multiplet with theoretical width of $0.6 \mathrm{eV}$, but the resolution of the XPS equipment is much lower. In Figure 8 we show XPS on semimetallic GdSb [3] [21] and in Figure 9 we show the same results on semiconducting, non-stoichiometric stretched films of GdN [10]. Whereas in Figure 8 the center of the peak is at $9 \mathrm{eV}$ it is only $7.8 \mathrm{eV}$ in Figure 9. The difference is apparently the position of the Fermi level which in GdSb is higher than in semiconducting GdN. The small peak starting at the Fermi level is the $2 p^{6}$ band of the anions. Unfortunately no XPS data exist on stoichiometric single crystals of GdN. But using also the absorptive dielectric constant $\sigma_{1}$ [3] [7] on single crystals we find the strongest oscillator strength for a $4 \mathrm{f}^{7}-5 \mathrm{dt}_{2 \mathrm{~g}}$ transition at about $9 \mathrm{eV}$.

For the magnetic exchange interaction we have two main contributions: according to Goodenough [22] we have a cation-cation superexchange with virtual transfer of a $4 \mathrm{f}$ electron to the $5 \mathrm{dt}_{2 \mathrm{~g}}$ excited state of a neighbor cation. In third order perturbation energy $J_{1}=t^{2} J_{f d} / 2 S^{2} U^{2}\left(f-d t_{2 g}\right)$, where $U\left(f-d_{2 g}\right)$ is the energy difference $4 \mathrm{f}^{7}-5 \mathrm{dt}_{2 \mathrm{~g}}$ band and $\mathrm{J}_{\mathrm{fd}}$ is the intra-atomic exchange constant of about $0.1 \mathrm{eV}$ [23]. The transfer integral $\mathrm{t}$ is estimated to be about $0.15 \mathrm{eV}$ [23]. Using the XPS value of about $9 \mathrm{eV}$ from Figure 8 we obtain for the ferromagnetic contribution $\mathrm{J}_{1}=0.07 \mathrm{~K}$, an extremely small value due to the large $4 \mathrm{f}-5 \mathrm{dt}_{2 \mathrm{~g}}$ separation. 

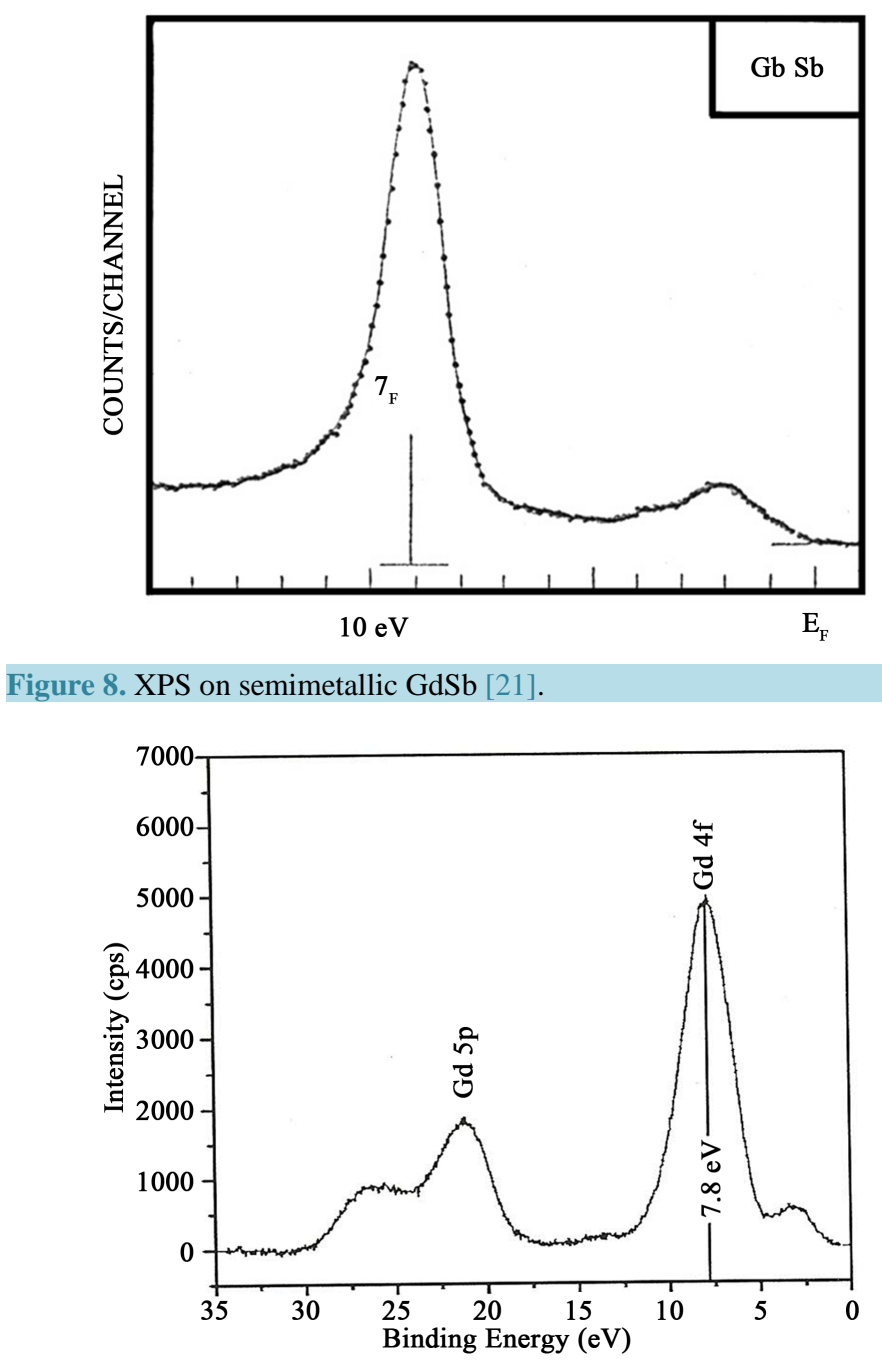

Figure 9. XPS on semiconducting GdN films [10].

For the antiferromagnetic exchange $\mathrm{J}_{2}$ an indirect superexchange has been assumed [23] involving a covalency factor and the energy difference $\mathrm{U}\left(\mathrm{p}-\mathrm{de}_{\mathrm{g}}\right.$ ), taken from Ref. [24] to be about $5.4 \mathrm{eV}$. Thus $\mathrm{J}_{2}$ turns out to be $\mathrm{J}_{2}$ $=-0.35 \mathrm{~K}$ [3] [7]. With such a small $\mathrm{J}_{1}$ and such a large and negative $\mathrm{J}_{2}$ the lattice contribution to the magnetic exchange is clearly antiferromagnetic. But this still corresponds to an insulating magnetic material. However, this is being claimed for the thin film results [10]-[16] but with molecular field equations $T_{N}=-63 J_{2}$ and we obtain $T_{N}=22 \mathrm{~K}$. Not bad, considering the experimental values from Figure 3 between 20 and $40 \mathrm{~K}$. Thus again, even for thin insulating films, when measured with magnetic fields in the order of 10 Oe and neglecting a RKKY interaction the result must be antiferromagnetism. But such measurements have not been performed.

However, when we consider the comparison between $\mathrm{EuO}$ and $\mathrm{GdN}$ as done in Ref. [16] we see in Ref [20] that with the same theory as above for $\mathrm{EuO} \mathrm{J}_{1}=+0.606 \mathrm{~K}$ and $\mathrm{J}_{2}=+0.119 \mathrm{~K}$, a clear cut ferromagnet. For the insulating antiferromagnet EuTe with $4 \mathrm{f}^{7} 2 \mathrm{eV}$ below the Fermi level, $\mathrm{J}_{1}=0.043 \mathrm{~K}$ and $\mathrm{J}_{2}=-0.15 \mathrm{~K}$, similar values as for GdN. These exchange interactions according to Goodenough [22] have been found valid for all rare earth chalcogenides [25] and are used therefore also for GdN. One observes, the larger the $4 \mathrm{f}^{7}$ separation from the Fermi level, the more antiferromagnetic are the samples. This holds especially also for semiconducting thin films which should be antiferomagnetic, but to our surprise are ferromagnetic.

However, when semimetallic and stoichiometric GdN single crystals are ferromagnetic (and this can be for excess electrons or high magnetic fields, probably even as low as 200 Oe) than this can happen only due to a second exchange contribution, namely RKKY of the free carriers. Now stoichiometric semimetallic single crys- 
tals of GdN have such free carriers, according to the measured Hall effect more than 6\% per Gd ion [18].

We measured the paramagnetic Curie Temperature $\Theta$ in function of carrier concentration and for this pupose we should use mixtures of GdN with GdO, because in GdO Gd is still trivalent and donates $15 \mathrm{~d}$ electron to the conduction band. But GdO does not exist. So we tried mixtures between GdP and GdS, both unquestioned antiferromagnets. This is shown in Figure 7 from about 10\% electrons per Gd Ion until 120\%. This was the first time that a complete RKKY oscillation could be measured. For low carrier concentration until about $20 \% \Theta$ is positive, i.e. ferromagnetic and for larger carrier concentration it is negative, i.e. antiferromagnetic. Since in GdN the carrier concentration is low its contribution is ferromagnetic and it can make the whole substance ferromagnetic. In order to check a net antiferromagnetism one must keep the carrier concentration as low as possible, and this can be achieved with stoichiometric GdN. Thus with $6 \%$ carriers their ferromagnetic contribution is not large enough to make the whole material ferromagnetic. But with already $8 \%$ carriers, as measured with the Hall effect, the material is from the beginning on ferromagnetic.

So we conclude that GdN with $6 \%$ carriers or less and low magnetic fields (10 Oe) is antiferromagnetic but becomes ferromagnetic with large magnetic fields. The term is metamagnetic. With more than $6 \%$ carriers the material is ferromagnetic from the beginning, also in low magnetic fields. This can be seen again in Figure 4, where the initial slope is linear with field and only in higher fields $\mathrm{GdN}$ becomes ferromagnetic. $\mathrm{GdN}_{0.75} \mathrm{O}_{0.25}$ with about $30 \%$ carriers is from the beginning on antiferromagnetic.

\section{Proposal with Magnetic Polarons}

It is then not clear how semiconducting GdN films, i.e. without significant carriers, can be ferromagnetic since the magnetic contribution from the lattice is antiferromagnetic. Another magnetic contribution must be active.

In comparing EuO with GdN (both with $4 \mathrm{f}^{7}$ and ${ }^{8} \mathrm{~S}_{7 / 2}$ ) it is remarkable that EuO as an $\mathrm{S}$ state does not show a hysteresis loop and has no coercive field. But ferromagnetic thin films do show a hysteresis loop as shown in Figure 10. This film had a lattice constant of $5.05 \AA, T_{C}=29 \mathrm{~K}$ and a saturation magnetization of only 5.6 $\mu_{\mathrm{B}} / \mathrm{Gd}$, but it was measured in a low field of only 20 Oe. The ordering temperature is far from the standard $70 \mathrm{~K}$ and is in the ball park of $\mathrm{T}_{\mathrm{N}}$ of the single crystals. This film is on the way to antiferromagnetism. Especially interesting is the existence of a hysteresis loop (also seen in other thin films of GdN) and a coercive field of about 100 Oe, inexistent on EuO. It shows that there are orbital moments involved. The short range ordering is significant and the correlation length is considered to be shorter than $30 \mathrm{~nm}$. But also other thin films are reported [14] which have ordering temperatures near $32 \mathrm{~K}$. But they all seem to be still ferromagnetic and semiconducting.

We have seen that in the discussion of the magnetism of stoichiometric single crystals the lattice contribution is antiferromagnetic due to the low lying $(9 \mathrm{eV}) 4 \mathrm{f}^{7}$ localized state. Ferromagnetism is only induced by the RKKY interaction which necessitates semimetallic behavior. How then can a semiconducting GdN be ferromagnetic? We have stated already in the chapter of preparation of thin films that these films in principle have a large number of voids in the lattice. The lattice constant of the films is generally $1.5 \%$ larger than in stoichiometric single crystals. Although an expansion of the lattice generally leads to semiconductors (to be discussed fur-

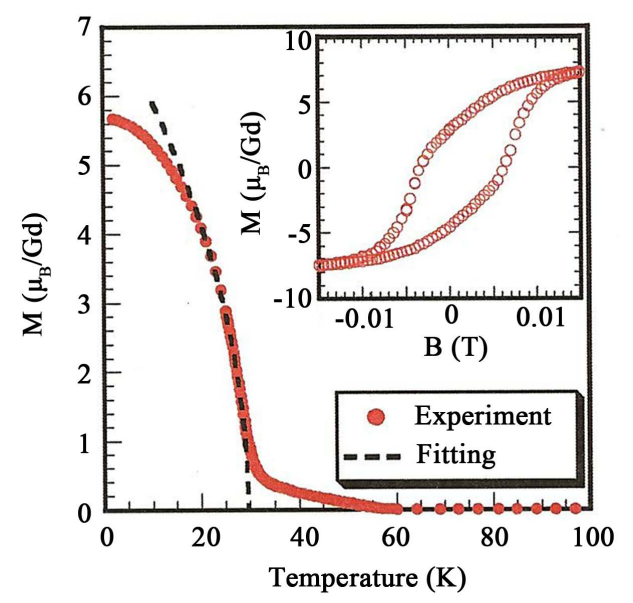

Figure 10. Magnetisation and hysteresis loop of a GdN film [15]. 
ther down) the lattice expansion in films is too small to provoke semiconductivity. We therefore must conclude that the existence of the voids is the essential parameter to create semiconductors. Since all the films have been prepared with excess nitrogen, sometimes in reactive sputtering [10] with nitrogen ions we may assume that the excess nitrogen finds its place in the voids. There it acts as an acceptor as it is three times ionized. These nitrogen ions absorb to a great part the electrons from the $5 d$ conduction band and provoke semiconductivity. As the electrons disappear from the $5 \mathrm{~d}$ conduction band simultaneously also the holes in the p-band of nitrogen become reduced and thus a small gap opens. The concentration of voids is generally much larger than the few percents charge carriers (see Figure 2) so some ionized nitrogen persist.

Here we can have the phenomenon of small (or large) magnetic polarons. These have been proposed already 1967 by Nagaev [28] and been the first time experimentally shown (1970) on EuSe by Streit and Wachter [29]. In GdN films there are thus still some charged acceptor nitrogen ions and a small amount of electrons in the 5d conduction band. These electrons circle the ionized nitrogen in a Bohr orbit including many Gd ions and with the spin of the $5 \mathrm{~d}$ electrons create a ferromagnetic micro-region-a magnetic polaron. These can be cooperative with other magnetic polarons when they are large, else wise ferromagnetic micro regions prevail. The $30 \mathrm{~nm}$ proposed in Ref. [15] seem reasonable. Also in Ref. [16] magnetic polarons have been proposed for ferromagnetism, but the authors propose an empty (but charged) vacancy.

So at the same time we can explain the semiconducting nature of the films and with magnetic polarons introduce the second magnet contribution, which we were looking for. Also the orbital contribution is present with the $5 \mathrm{~d}$ magnetic polaron and thus a hysteresis loop can exist. On the other hand we must insist, that GdN films do not represent stoichiometric GdN. There is thus the world of stoichiometric (tested) single crystals, which are antiferromagnetic semimetals for low magnetic fields and in larger fields or with excess electrons are ferromagnetic. And the world of thin films which are semiconductors and ferromagnetic due to magnetic polaron effects.

\section{Optical Properties}

We can continue with the confrontation of stoichiometric single crystals of GdN and thin films of GdN for optical results. In Figure 11 we show the reflectivity of GdN single crystals between $30 \mathrm{meV}$ and $13 \mathrm{eV}$. The plasma edge of the free carriers is clearly visible in the infrared and corresponds to about $6 \%$ carriers pro Gd and the optical effective mass is $\mathrm{m}_{\text {oeff }}=0.8 \mathrm{~m}_{\mathrm{e}}$, appreciable larger than in nonmagnetic $\mathrm{ScN}$, where it is $\mathrm{m}_{\text {oeff }}=0.13 \mathrm{~m}_{\mathrm{e}}$. With 6\% free carriers single crystals are not simply a doped material, but it is a semimetal, due to the indirect overlap of $2 \mathrm{p}(\Gamma)$ and $5 \mathrm{~d}(\mathrm{X})$ (see below). In Figure 12 we show the absorptive part $\sigma_{1}$, obtained after a Kramers-Kronig transformation of the reflectivity of Figure 11. However, thin films show an absorption edge and gap at about $1.2 \mathrm{eV}$ (the same as in EuO [20]) and only a small Drude like free carrier absorption in Figure 13 [15]. The films are semiconducting [10]-[16].

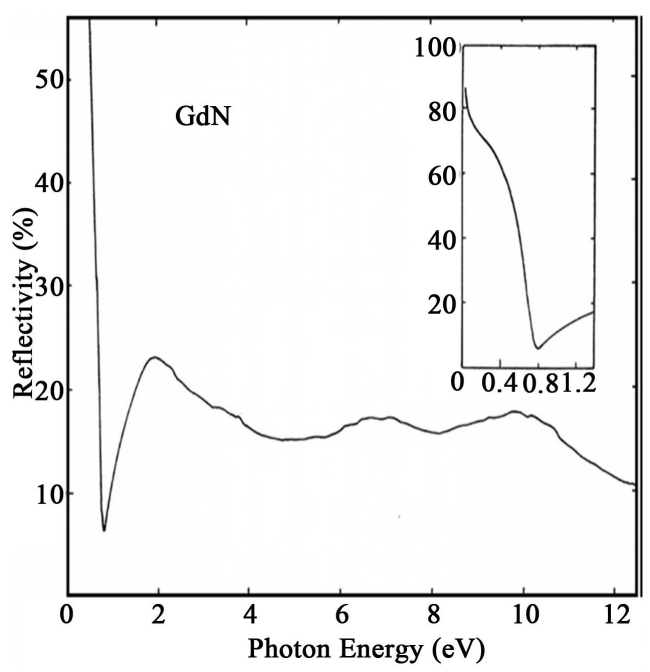

Figure 11. Optical reflectivity between $30 \mathrm{meV}$ and $13 \mathrm{eV}$ on $\mathrm{GdN}$ single crystals [3] [7]. 


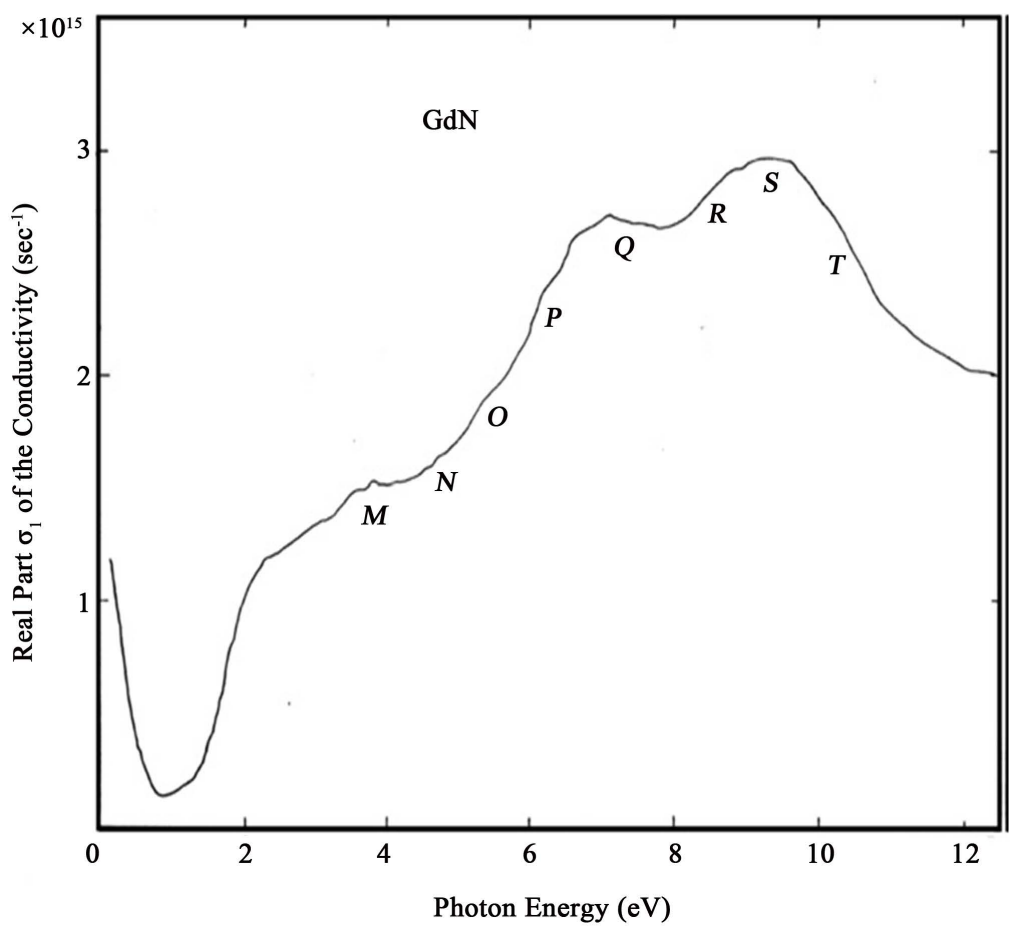

Figure 12. Absorptive part of dielectric constant of single crystal of GdN [3] [7].

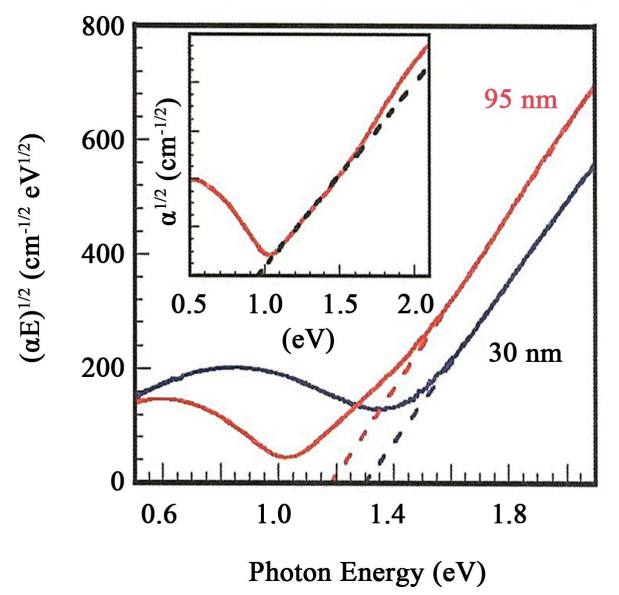

Figure 13. Absorption of a 30nm and $95 \mathrm{~nm}$ thin film of GdN [15].

In Figure 14 we observe a shift of the plasma edge of Figure 11 with temperature. We recollect that single crystals in zero magnetic field are antiferromagnetic and semimetallic. Lowering the temperature from $300 \mathrm{~K}$ to about $70 \mathrm{~K}$ we observe a blue shift of the plasma edge which is due to the thermal contraction with temperature. But below $70 \mathrm{~K}$ an additional blue shift due to the antiferromagnetic ordering is observed, just as in antiferromagnetic EuTe [20]. But when we apply a magnetic field of $1.2 \mathrm{~T}$ and make the crystal ferromagnetic a red-shift is observed just like in ferromagnetic EuO [20]. This effect is due to the exchange splitting of the 5d conduction band and it has the form of a spin correlation function just as in EuO.

On the other hand for GdN films the absorption edge also makes a red-shift upon ferromagnetic ordering, as shown in Figure 15. The amount of this shift is with $270 \mathrm{meV}$ exactly the same as for ferromagnetic EuO [20]. Also others measured a ferromagnetic red-shift on films of GdN [30].

Again we have the contrast of antiferromagnetic and metallic single crystals and ferromagnetic semiconductors in films. But both have a red-shift due to the exchange splitting of the $5 \mathrm{~d}$ band upon ferromagnetic order, 


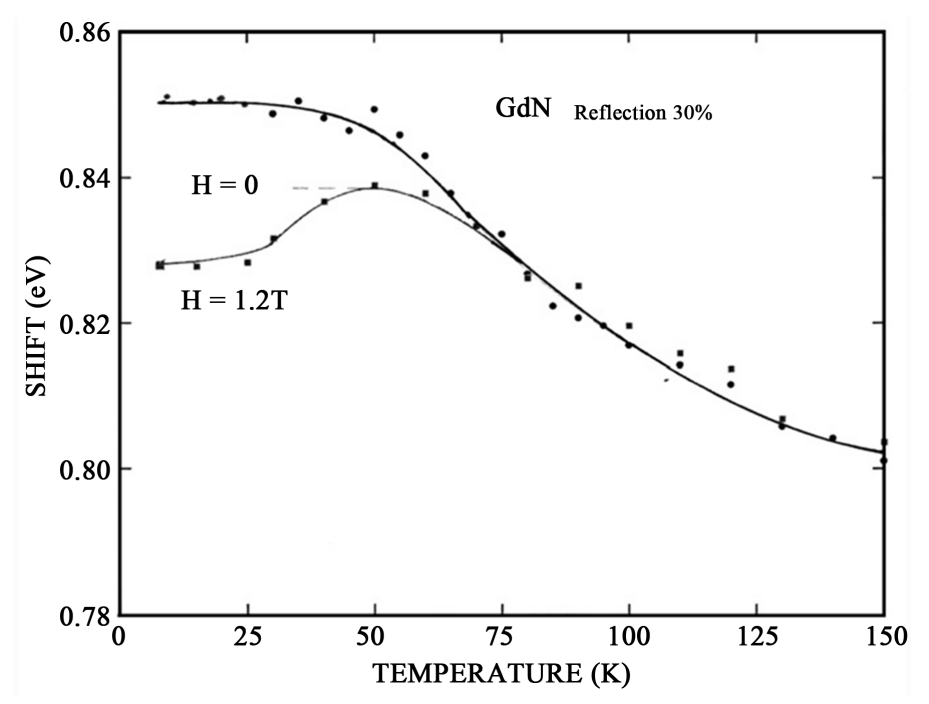

Figure 14. Shift of the plasma edge of GdN single crystals in function of temperature and magnetic field [3] [7].

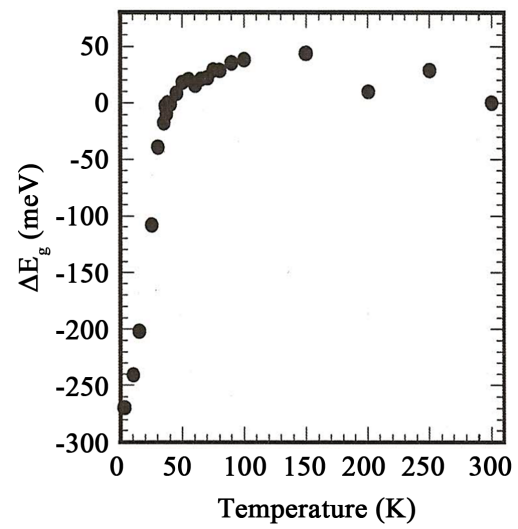

Figure 15. Shift of the absorption edge of ferromagnetic GdN films [15].

somewhat smaller in GdN crystals than in films due to the screening effect of the significant charge carriers in GdN crystals.

\section{Electric Transport Properties.}

The contrast between stoichiometric single crystals and non-stoichiometric films of GdN can be continued with transport properties. At room temperature we have in single crystals a negative Hall effect of $\mathrm{n}=1.9 \times 10^{21} \mathrm{~cm}^{-3}$ [3] [7] [18] and in films about $\mathrm{n}=4 \times 10^{20} \mathrm{~cm}^{-3}$ [12]. The electrical resistivity was in single crystals $\rho=4 \times 10^{-4}$ $\Omega \cdot \mathrm{cm}$ [3] [7] and in films $0.3 \Omega^{\prime} \Omega \cdot \mathrm{cm}$ [13]. The mobility $\mu=(\rho \text { ne })^{-1}$ was $\mu=8 \mathrm{~cm}^{2} /$ Vsec in single crystals and only $0.05 \mathrm{~cm}^{2} /$ Vsec in thin films [13]. Such a small mobility in films is due to the inhomogeneity of the films, the voids or vacancies and the spin fluctuations at $300 \mathrm{~K}$. It shows that the films are in a terrible state. Drastical differences are observed in the temperature dependence of the resistivity. In Figure 16 we plot the temperature dependence of GdN single crystals which is clearly metallic and for $\mathrm{H}=0$ looks the same as for GdP, GdAs, GdSb and GdBi [31] with a little hump at the magnetic ordering temperature of about $40 \mathrm{~K}$. In a magnetic field of $1 \mathrm{~T}$, when the crystals are ferromagnetic we observe already an increase in the resistivity with a saturation for the lowest temperatures. This is in agreement with the spin correlation function of the exchange splitting of the $5 \mathrm{~d}$ conduction band [3] [7] and it is compatible with Figure 14. The red-shift of the plasma edge upon ferromagnetism is due to a reduction of charge carriers and thus an increase of resistivity as in Figure 16.

In contrast we show in Figure 17 the temperature dependence of the electrical resistivity of GdN films which 


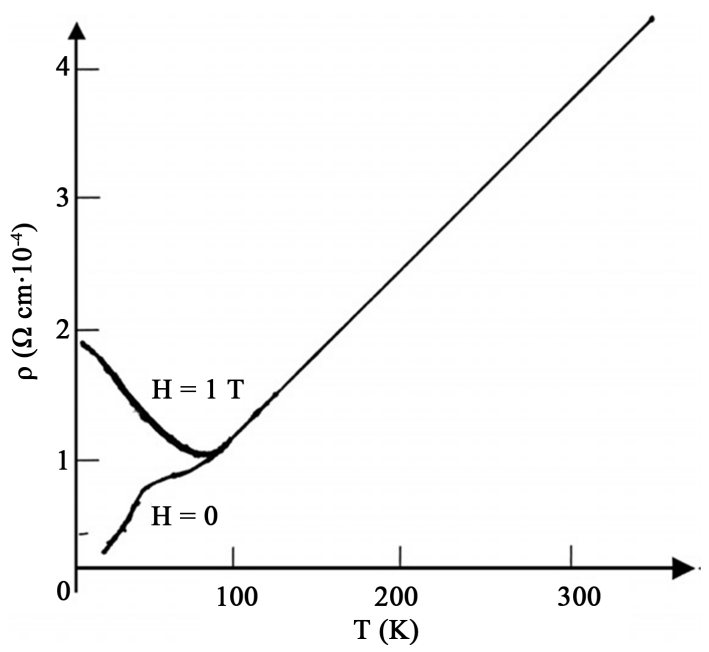

Figure 16. Electrical resistivity of GdN single crystals [3] [7].

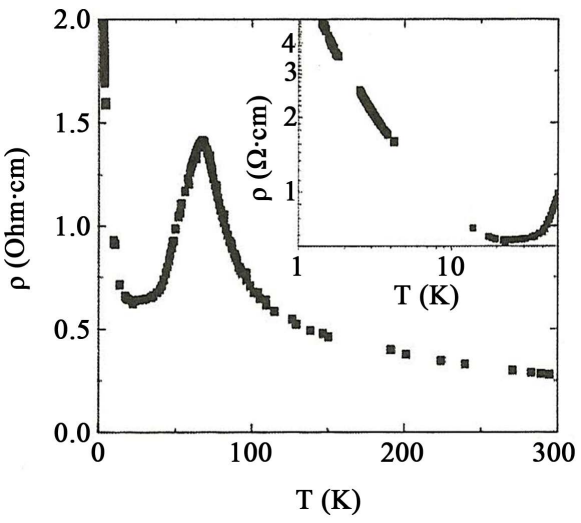

Figure 17. Temperature dependence of the resistivity of GdN films [13].

exhibit a significant maximum at the Curie temperature of about $70 \mathrm{~K}$ but below, shown in the inset of Figure 17, continues to rise at the lowest temperatures, but only with an activation energy of $5 \mathrm{meV}$ [13]: clearly a semiconducting behavior, although the activation energy is only in the meV. It corresponds to the binding energy of the magnetic polarons and not to the semiconductor gap.

And so it can go on. Clearly experimentally there are two worlds: the one of stoichiometric (tested by Kjeldahl method) single crystals and those of non-stoichiometric thin films with lots of voids or vacancies. The experimental results within one world are consistent inasmuch as single crystals are antiferromagnetic in fields of only 10 Oe, when they have a small carrier concentration below 6\% of the Gd concentration and they are ferromagnetic in higher magnetic fields, they are semimetals, have a pronounced plasma edge in optical reflectivity, but show also the magnetic red-shift when ferromagnetic and in function of a magnetic field. Thin films are ferromagnetic from the beginning not due to higher carrier concentration because they are semiconducting. But due to the voids they have nitrogen ions in the voids which act as positive acceptors, suck up the conduction carriers in the conduction band and create magnetic polarons, which in turn make the films ferromagnetic. Optically they have an absorption edge which shifts to the red when ferromagnetic, just as in EuO [20].

But do thin films represent stoichiometric GdN? Certainly not and their ferromagnetism and semiconducting behavior is due to defects in the two-dimensional lattice.

\section{Band Structure Considerations}

The basic problem of band structure calculation in rare earths and their compounds is the simultaneous presence of localized $4 \mathrm{f}$ states and broad bands. These states cannot be treated on the same footing. Always the localized 
4f states come out as narrow bands. It is therefore feasible to perform a band structure calculation for a "near" rare earth nitride without $4 \mathrm{f}$ states, such as $\mathrm{ScN}$ to get a feeling how $2 \mathrm{p}^{6}$ anion bands and $5 \mathrm{~d}$ bands are interacting. So in a self consistent density-functional ground-state formalism the band structure for ScN has been calculated [32] and is shown in Figure 18. In an optical reflectivity study from $1 \mathrm{meV}$ to $13 \mathrm{eV}$ photon energy and with evaluation of the dielectric constants from a Kramers-Kronig relation [33] all the optical transitions between equal grad $\mathrm{E}(\mathrm{k})$ initial and final states of the optical transition could be verified experimentally [7] [33].

In Figure 19 a local spin density approximation LSDA + U with Hubbard U has been used to calculate the band structure of GdN films [30]. The exchange splitting of the bands is shown in Figure 19 with dashed lines, but this is not relevant for GdN in its single crystalline form since it is not ferromagnetic in the ground state but an antiferromagnet (see above). The arbitrary chosen $\mathrm{U}=8 \mathrm{eV}$ takes its value from Figure 9, where it is $7.8 \mathrm{eV}$. It is remarkable that the dispersion of $\mathrm{p}$ and $\mathrm{d}$ bands for $\mathrm{GdN}$ films in Figure 19 represent exactly the $\mathrm{p}$ and $\mathrm{d}$ bands in Figure 18 for ScN.

The big and significant difference between Figure 18 and Figure 19 is that the band structure for ScN yields an indirect overlap of $60 \mathrm{meV}$ between $\mathrm{p}(\Gamma)$ and $\mathrm{dt}_{2 \mathrm{~g}}(\mathrm{X})$ and results thus in a semimetal, whereas in Figure 19 for GdN films an indirect gap of about $1 \mathrm{eV}$ has been assumed (not calculated), taken from optical experiments [15] [30]. The measured optical gap of semiconducting GdN films of about $1 \mathrm{eV}$ has been introduced in the calculation and (I admit) I can do this also by hand and shift the $5 \mathrm{dt}_{2 \mathrm{~g}}$ band by that amount.. Thus the calculation of Ref. 30 uses three experimental parameters: the lattice constant of $5.05 \AA$, the Hubbard U of $8 \mathrm{eV}$ and the optical gap of $1 \mathrm{eV}$. It is not self consistent. I remember the famous statement of Phil Anderson: "with three parameters I can fit an elephant". In a GdN single crystal the lowest energy optical transition is between $\mathrm{p}(\mathrm{X})$ and $\mathrm{dt}_{2 \mathrm{~g}}(\mathrm{X})$ and it corresponds with $1.3 \mathrm{eV}$ to the direct gap (see Figure 12). But due to the indirect overlap of $\mathrm{p}(\Gamma)$ and $\mathrm{dt}_{2 \mathrm{~g}}(\mathrm{X})$ it is still a semimetal.

In fact there are quite a number of band structure calculations for GdN (and other rare earth nitrides) yielding a semimetal with indirect $\mathrm{p}(\Gamma)$ and $\mathrm{d}(\mathrm{X})$ overlap or an indirect gap between $\mathrm{p}$ and $\mathrm{d}$ bands yielding semiconductors. I refrain from quoting all of them, but use for comparison and examples only the band structure of semiconducting GdN films [30] and semimetallic GdN [24].

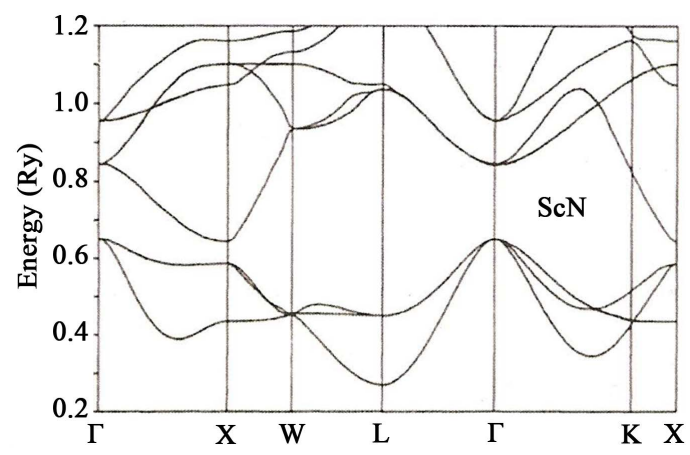

Figure 18. Band structure of ScN [32].

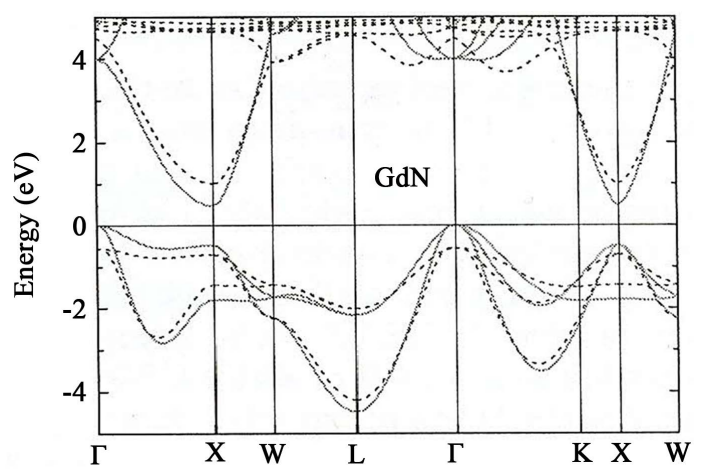

Figure 19. Semiempirical band structure of GdN [30] with $d$ bands shifted upwards by hand. 
In Figure 20(a) there is no Hubbard $U$ involved and as a consequence the occupied $4 \mathrm{f}^{7}$ states are strongly hybridized with the nitrogen $2 \mathrm{p}$ band, which is physically incorrect. But the dispersion of the $2 \mathrm{p}^{6}$ and the $5 \mathrm{dt}_{2 \mathrm{~g}}$ bands are practically the same as in ScN [32], especially there is an indirect overlap of $2 p(\Gamma)$ and $5 \mathrm{~d}(\mathrm{X})$, again just as in ScN [32], making the material a semimetal. The exchange splitting of the bands due to ferromagnetism is obsolete, since the ground state is not ferromagnetic but antiferromagnetic as discussed above. But with a relatively strong magnetic field GdN becomes ferromagnetic and then the exchange splitting is in the right order of magnitude (see also the "red-shift" above).

In Figure 20(b) a Hubbard $U_{\text {eff }}=9.2 \mathrm{eV}$ is introduced, which does not change the $\mathrm{p}$ and $\mathrm{d}$ bands but shifts the occupied $4 \mathrm{f}^{7}$ states to $7.5 \mathrm{eV}$ below the Fermi energy. In reality it should be about $9 \mathrm{eV}$ below the Fermi energy (see Figure 8). Again the exchange splitting due to ferromagnetism is only correct in single crystals in an applied magnetic field and the distinction into spin majority and minority bands does not exist in the ground state and as a consequence the material is not a half metal. But generally speaking the band structure of Figure 20(b) is quite convincing especially the calculation of a semimetal, as found experimentally on stoichiometric single crystals. Also the equilibrium lattice constant as the energy minimum of the crystal is with $4.974 \AA$ very close to the measured value of $4.984 \AA$. The semimetal has of course a hole pocket at the $\Gamma$ point and an electron pocket at the $\mathrm{X}$ point of equal size, but the Hall effect is negative, i.e. the electrons are dominant.

But as a simple minded experimentalist the question may be permitted: why is the same type of calculation LSDA + U leading to a completely different band structure, in one case a semiconductor with an indirect gap of about $1 \mathrm{eV}(\mathrm{GdN}$ films), and in the other case to a semimetal with an indirect overlap of $\mathrm{p}(\Gamma)$ and $\mathrm{d}(\mathrm{X})(\mathrm{GdN}$ single crystals)?

As we have seen above, all GdN films are non-stoichiometric and have lattice constants always above $5.0 \AA$, sometimes up to $5.12 \AA$ [14] compared with the stoichiometric single crystals of $4.984 \AA$. Since it is well established that under hydrostatic pressure GdN becomes more metallic [34] one may ask the question whether a lattice expansion as observed in GdN films may lead to semiconductors? Theorists can make this answer quite easy, since for them an increase of the lattice constant is only a click with the computer. This has been done [35], but the lattice parameter has to be increased to more than $5.63 \AA(+14 \%)$ to provoke a gap between $\mathrm{p}(\Gamma)$ and $\mathrm{d}(\mathrm{X})$. In other words the increased lattice constant in the GdN films compared with the one on single crystals is not the reason for GdN films to become semiconductors. It are the voids filled with nitrogen ions which increase the lattice constant in the films and which suck away charge carriers from the conduction band.

The exchange parameters $J_{1}$ and $J_{2}$ have also been evaluated from the band structure [24] [35]. However, an error occurred in Ref. [35] as the scale in their Figure 3 is incorrect with $J_{1}=-0.86 \mathrm{meV}$ and $\mathrm{J}_{2}=+0.14 \mathrm{meV}$. The correction is in Ref. [24] with $\mathrm{J}_{1}=+0.86 \mathrm{meV}$ and $\mathrm{J}_{2}=-0.14 \mathrm{meV}$. The authors of Ref. [24] try to separate a super exchange contribution from the lattice, which they find to be antiferromagnetic and a ferromagnetic contribution from the free carriers in the sense of a RKKY interaction. These contributions are the same as have

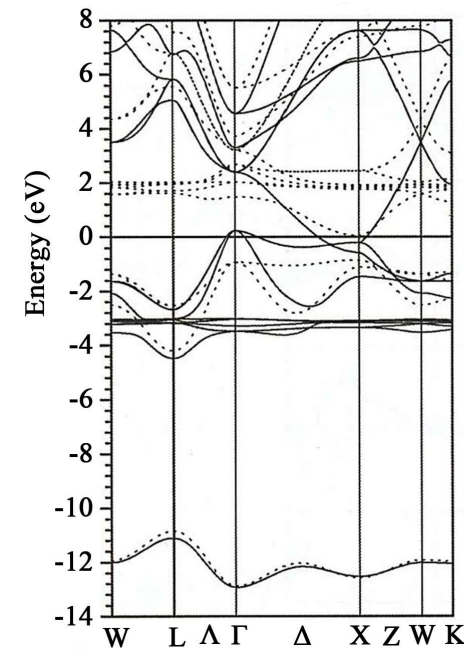

(a)

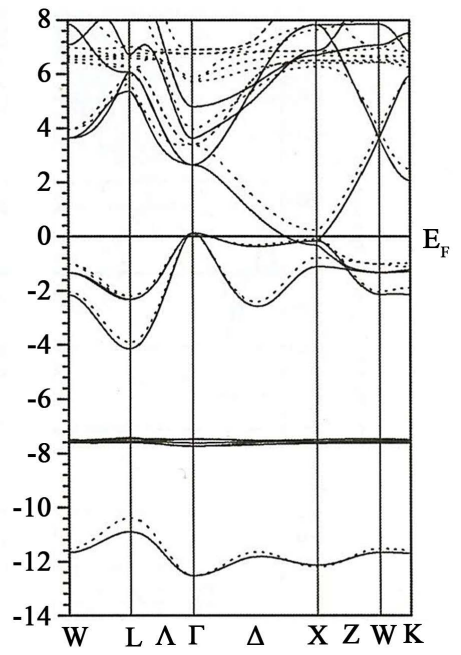

(b)

Figure 20. Self consistent band structure of GdN. (a) LSDA; (b) LSDA + $\mathrm{U}_{\text {eff }}$ [24]. 
been assumed in Refs. [3] [7], but the exchange parameters derived above in chapter 5 on magnetic exchange interactions are only for the super exchange interactions of the lattice and do not compare directly with the $\mathrm{J}_{1,2}$ derived for the total contribution of super exchange and RKKY.

In Ref. [24] many possibilities of increasing $T_{C}$ in GdN by alloying, doping or introducing defects are discussed. This is generally true, but these effects always result in ferromagnetic metals. These are of no interest since ferromagnetic metals exist in abundance already. New ferromagnetic semiconductors are a challenge, especially those with high $\mathrm{T}_{\mathrm{C}}$. And for applications ferromagnetic and semiconducting $\mathrm{EuO}$ is still the best, doped with Gd the Curie temperature increases up to $129 \mathrm{~K}$ [20]. But since more than 50 years there are practically no applications.

\section{Discussion}

It is quite clear that GdN is at the crossroad between ferro-and antiferromagnetism and between semimetal or semiconductor. From the beginning on and for polycrystalline material there was always an excess of metal for the nitrides, especially for GdN (literature in part in Ref. [3] [7] but the material was always of pure rocksalt structure. The material also behaved metallic in its temperature-resistivity behavior. The question, which was put very early was: is this metallicity a consequence of non-stoichiometry and Gd excess or is due to the band structure with band overlap. So in this laboratory there was a gradual progress from polycrystal and non-stoichiometric material over polycrystal stoichiometric material towards non-stoichiometric single crystals to stoichiometric single crystals and of adequate size. A process over many decades and investments of several million dollars (not counting the salaries of the scientists). Also the development of analytic methods such as the Kjeldahl method and finally a micro-Kjeldahl method [8] [9], which permitted the chemical analysis of even one single crystal. In Figure 1 we show the tungsten vessels for the crystal growth and several GdN single crystal with size up to $5 \mathrm{~mm}$. The methods to handle the crystals and transfer them without air contact to the various experiments is described above [3].

Instead the growth of thin films suffered from the impossibility of determining the stoichiometry, and if such methods have been used, from the precision of the methods to fractions of percents. Besides, thin films always need substrates, very often sapphire and a protection against oxidation, usually $\mathrm{GaN}$ or $\mathrm{MgF}_{2}$ [10]-[16]. If the films would be stoichiometric they would be stable in air, just like the surface of stoichiometric single crystals [3] [7]. Optical measurements thus need huge correction processes, which are not always reasonable. The method to prepare thin films was everywhere the same [10]-[16], namely to co-evaporate a Gd film with excess nitrogen on a substrate. For principal reasons two-dimensional films always have voids or vacancies (Mandelbrot pattern) and these are demonstrated in Figure 2. One can discuss whether these voids are empty but charged [16] or filled with the excess nitrogen. In both cases a magnetic polaron ferromagnetism is valid. It has been described above that there is a more successful process to create thin films, namely the preparation of GdN stoichiometric polycrystals and then evaporate them on a substrate material. It is a big difference to condense GdN molecules compared to the condensation of Gd metal, which shall then be nitrided.

Since the exchange mechanism calculated for the super exchange of the ions for single crystals alone always gave antiferromagnetism because the $4 \mathrm{f}^{7}$ level was at so low energies, it was only the RKKY interaction of the free carriers, which could lead to ferromagnetism. In stoichiometric single crystals the carrier concentration could be reduced compared to non-stoichiometric material, so that with $6 \%$ carriers compared with the Gd concentration the RKKY was so much reduced that the compound as a whole was antiferromagnetic. But with already $8 \%$ carriers the crystals where ferromagnetic from the beginning and not only by application of a magnetic field.

In thin films, however, ferromagnetism prevailed (although with reduced saturation moment). Since also here the super exchange mechanism of the lattice was antiferromagnetic, but no free carriers were available as RKKY because the material also was semiconducting, another ferromagnetic contribution must be dominant. There the voids or vacancies in the two dimensional lattice acted as charged acceptors, either empty or filled with excess nitrogen ions, trapping remaining electrons, which circled the voids in a Bohr-like orbit containing hundreds of Gd ions and aligning their magnetic moments parallel to the one of the electrons, creating so ferromagnetic microregions. This phenomenon is well known and called a magnetic polaron. This then makes the films ferromagnetic, but with reduced saturation moment (see Figure 10).

It is then clear that the thin films are defect dominated and do not represent GdN in its pure and perfect state. 
One of the deadly arguments is the measurement of the electric mobility. In ScN single crystals it has been determined to be $260 \mathrm{~cm}^{2} / \mathrm{Vsec}$ [33] and it comes only from electron-phonon scattering, since magnetic moments are absent. In GdN single crystals the mobility is only $8 \mathrm{~cm}^{2} / \mathrm{Vsec}$, because at room temperature we now have in addition to phonon scattering also spin flip scattering which reduces the mobility [3] [7]. But in GdN films the mobility is further dramatically reduced to $0.05 \mathrm{~cm}^{2} / \mathrm{Vsec}$ [13], which is so small that one can think of electron hopping. Of course now, in addition there is impurity or defect scattering, dominating the resistivity behavior.

It then is a fact that there are two worlds of GdN: stoichiometric single crystals, which are metallic, in magnetic fields of about 10 Oe are antiferromagnets, but with excess electrons become ferromagnetic, just as with an external magnetic field. They achieve the saturation magnetisation of $7 \mu_{\mathrm{B}}$ in reasonable magnetic fields of about $10 \mathrm{kOe}$. In optical experiments one observes a huge plasma resonance due to the free carriers and various magneto-optical effects of shifts of this plasma resonance with the ferromagnetic order. A so called "red-shift of the plasma edge is observed upon ferromagnetic order. The experimental properties of single crystals are in itself consistent. Theoretical band structure calculations confirm the magnetic and electronic behavior due to an indirect band overlap of $2 \mathrm{p}^{6}(\Gamma)$ with $5 \mathrm{dt}_{2 \mathrm{~g}}(\mathrm{X})$. The LSDA + U calculation [24] yields for $\mathrm{p}$ and $\mathrm{d}$ bands the same band structure as in ScN [32] and the U or $\mathrm{U}_{\text {eff }}$ (taken from XPS) takes care of the $4 \mathrm{f}^{7}$ localized level. Exchange splitting and half metallicity are absent in the antiferromagnetic ground state.

The world of thin films suffers from the fact that every group of authors has produced different thin films (10 - 16), but they all agree in the fact that they are ferromagnetic and semiconducting in the ground state. They have all larger lattice constants when compared with poly-or single crystals, but the $1.5 \%$ larger lattice constants in the average are not sufficient to create semiconductivity [35]. This is the effect of the voids which trap excess nitrogen ions and in turn free electrons. The measured activation energy of the resistivity is with 5 meV the binding energy of the electrons onto the acceptors in the voids [13]. The optical experiments show an indirect gap of about $1 \mathrm{eV}$ and a direct gap of about $1.3 \mathrm{eV}$ [15] and exhibit the "red-shift" of about $270 \mathrm{meV}$ just as in $\mathrm{EuO}$ [20], but they do not have the complete saturation moment of $7 \mu_{\mathrm{B}}$. In itself the experiments are consistent. The band structure calculation [30] shows again the same 2p and $5 \mathrm{~d}$ bands as in $\mathrm{ScN}$, but the $5 \mathrm{~d}$ bands are hand shifted higher so as to give a gap of about $1 \mathrm{eV}$. Such a band structure calculation is useless and is not self consistent.

Without any doubt, the films are interesting, but not representative for GdN.

\section{Conclusions}

If one considers the whole spectrum of experimental and theoretical papers for GdN one observes that the material can be grown as stoichiometric single crystals or non-stoichiometric thin films. The growth chemistry is different in both cases. As a consequence the physical parameters differ in a fundamental way.

Stoichiometry and chemically tested single crystals are antiferromagnetic in their ground state, but can be made ferromagnetic with excess carriers or a strong magnetic field. The terminology is therefore metamagnetism. The carrier concentration is in the percentage range of the Gd concentration. The materials are semimetals as confirmed by Hall effect, electrical resistivity and plasma edge in optical experiments. They show the redshift in optical measurements which is a sign of exchange splittings of the conduction band upon ferromagnetic order. All experiments for single crystals support this conclusion.

Thin films due to their preparation method of co-evaporation of Gd metal in an excess nitrogen atmosphere are deposited on a substrate of different crystal structure and lattice constant and covered with another film of different crystal structure and lattice constant. The films are grown at low temperature, mostly room temperature and have a sizable amount of defects, voids or vacancies. They are electrically charged and can trap the percentage large carriers in the conduction bands and thus provoke semiconductivity. The observed ferromagnetism is due to the formation of magnetic polarons, electrons which circle charged acceptors in a Bohr like orbit of hundreds of Gd ions aligning all spins parallel, creating thus ferromagnetic micro regions. These cooperate in the case of large magnetic polarons. The thin films are defect dominated and do not represent stoichiometric material.

It is thus no surprise that there are numerous theoretical band structure calculations either for ferromagnetic semimetals or for ferromagnetic semiconductors and in each case they find experimental results which support their theory. However, it is not reasonable that the same type of calculation, the LSDA + U yields different band structures, such as for semimetals or semiconductors. 
This epic discussion will only stop when thin films can be grown with equal perfection as for single crystals. Although we have described a successful method for this above, nobody has yet made use of this.

\section{Acknowledgements}

The author is most grateful to Prof. Dr. Emmanuel Kaldis, who developed with his group during his time at the Laboratorium für Festkörperphysik at ETH Zürich the growth of large and well defined stoichiometric single crystals of Rare Earth Nitrides.

Valuable discussions with Prof. B. Batlogg and Prof. R. Monnier are gratefully acknowledged.

\section{References}

[1] Hulliger, F. (1979) Rare Earth Pnictides. In: Gschneidner Jr., K.A. and Eyring, L., Eds. Handbook on the Physics and Chemistry of Rare Earths, Vol. 4, North-Holland Publishing, Amsterdam, 153-236.

[2] Vogt, O. and Mattenberger, K. (1993) Magnetic Measurements on Rare Earth and Actinide Monopnictides and Monochalcogenides. In: Gschneidner Jr., K.A., Eyring, L., Lander, G.H. and Choppin, G.R., Eds., Handbook on the Physics and Chemistry of Rare Earths, Physics-I, Vol. 17, Lanthanides/Actinides, North-Holland Publishing, Amsterdam, 14407.

[3] Wachter, P. (2015) Physical Properties of some Stoichiometric Rare Earth Nitride Single Crystals. Advances in Materials Physics and Chemistry, 5, 96-131. http://dx.doi.org/10.4236/ampc.2015.53013

[4] Hirayama, Y., Nakagawa, T. and Yamamoto, T.A. (2011) Curie Temperatures and Modified de Gennes Factors of Rare Earth Nitrides. Solid State Communications, 151, 1602-1604. http://dx.doi.org/10.1016/j.ssc.2011.07.021

[5] Child, H.R., Wilkinson, M.K., Cable, J.W., Koehler, W.C. and Wollan, E.O. (1963) Neutron Diffraction Investigation of the Magnetic Properties of Compounds of Rare Earth Metals with Group V Anions. Physical Review, 131, 922-931. http://dx.doi.org/10.1103/PhysRev.131.922

[6] Sclar, N. (1964) Properties of Rare Earth Nitrides. Journal of Applied Physics, 35, 1534-1538. http://dx.doi.org/10.1063/1.1713662

[7] Wachter, P. (2012) Physical Properties of Stoichiometric GdN Single Crystals. Results in Physics, 2, 90-96. http://dx.doi.org/10.1016/j.rinp.2012.07.003

[8] Kaldis, E. (1974) Principles of the Vapour Growth of Single Crystals. In: Goldman, C.H.L., Ed., Crystal Growth: Theory and Techniques, Vol. 1, Plenum Press, New York, 49-192. http://dx.doi.org/10.1007/978-1-4757-1272-8 2

[9] Kaldis, E. and Zürcher, Ch. (1976) Solid State Chemistry and Physics of the Monopnictides and Monochalcogenides of Some Trivalent Rare Earths. In: Lundin, C.E., Ed., Proceedings of the 12th Rare Earth Research Conference, Denver Research Inst., Denver, 915-934.

[10] Leuenberger, F., Parge, A., Felsch, W., Fauth, K. and Hessler, M. (2005) GdN Thin Films: Bulk and Local Electronic and Magnetic Properties. Physical Review B, 72, Article ID: 014427. http://dx.doi.org/10.1103/PhysRevB.72.014427

[11] Azeem, M. (2015) preprint.

[12] Ludbrook, B.M., Farrell, I.L., Kuebel, M., Ruck, B.J., Preston, A.R.H., Trodahl, H.J., Ranno, L., Reeves, R.J. and Durbin, S.M. (2009) Growth and Properties of Epitaxial GdN. Journal of Applied Physics, 106, 063910. http://dx.doi.org/10.1063/1.3211290

[13] Granville, S., Ruck, B.J., Budde, F., Koo, A., Pringle, D.J., Kuchler, F., Preston, A.R.H., Housden, D.H., Lund, N., Bittar, A., Williams, G.V.M. and Trodahl, H.J. (2006) Semiconducting Ground State of GdN Thin Films. Physical Review B, 73, 235335. http://dx.doi.org/10.1103/PhysRevB.73.235335

[14] Khazen, K., von Bardeleben, H.J., Cantin, J.L., Bittar, A., Granville, S., Trodahl, H.J. and Ruck, B.J. (2006) Ferromagnetic Resonance Study of GdN Thin Films with Bulk and Extended Lattice Constants. Physical Review B, 74, 245330. http://dx.doi.org/10.1103/PhysRevB.74.245330

[15] Yoshitomi, H., Kitayama, S., Kita, T., Wada, O., Fujisawa, M., Ohta, H. and Sakurai, T. (2011) Optical and Magnetic Properties in Epitaxial GdN Thin Films. Physical Review B, 83, 155202. http://dx.doi.org/10.1103/PhysRevB.83.155202

[16] Natali, F., Ruck, B.J., Trodahl, H.J., Le Binh, D., Vezian, S., Damilano, B., Cordier, Y., Semond, F. and Meyer, C. (2013) Role of Magnetic Polarons in Ferromagnetic GdN. Physical Review B, 87, 035202. http://dx.doi.org/10.1103/PhysRevB.87.035202

[17] Wachter, P. (1972) The Optical, Electrical and Magnetic Properties of the Europium Chalcogenides and Rare Earth Pnictides. CRC Critical Reviews in Solid State Sciences, 3, 189-241. http://dx.doi.org/10.1080/10408437208244865

[18] Wachter, P. and Kaldis, E. (1980) Magnetic Interaction and Carrier Concentration in GdN and in GdN $\mathrm{H}_{1-\mathrm{x}} \mathrm{O}_{\mathrm{x}}$. Solid State 
Communications, 34, 241-244. http://dx.doi.org/10.1016/0038-1098/(80)90401-9

[19] Wachter, P. (1978) Electronic Structure, Magnetic Exchange and Electrical Transport Properties of the Magnetic Compoundfs EuS, GdS and GdP. Physics Reports, 44, 159-186. http://dx.doi.org/10.1016/0370-1573(78)90001-7

[20] Wachter, P. (1979) Europium Chalcogenides: EuO, EuS, EuSe and EuTe. In: Gschneidner Jr., K.A. and Eyring, L., Eds., Handbook on the Physics and Chemistry of Rare Earths, Vol. 2, Ch. 19, North-Holland Publishing, Amsterdam, 507-574.

[21] Campagna, M., Wertheim, G.K. and Baer, Y. (1979) Unfilled Inner Shells: Rare Earths and their Compounds. In: Ley, L. and Cardona, M., Eds., Topics in Applied Physics, Vol. 27, Photoemission in Solids II, Case Studies, Springer Verlag, Berlin, 217-260. http://dx.doi.org/10.1007/3-540-09202-1_4

[22] Goodenough, J.B. (1963) Magnetism and the Chemical Bond. Interscience Monographs on Chemistry, Vol. 1, John Wiley, New York, 1-385.

[23] Kasuya, T. and Yanase, A. (1968) Anomalous Transport Phenomena in Eu-Chalcogenide Alloys. Review of Modern Physics, 40, 684-695. http://dx.doi.org/10.1103/RevModPhys.40.684

[24] Duan, C.-G., Sabirianov, R.F., Mei, W.N., Dowben, P.A., Jaswal, S.S. and Tsymbal, E.Y. (2007) Electronic, Magnetic and Transport Properties of Rare Earth Monopnictides. Journal of Physics: Condensed Matter, 19, Article ID: 315220. http://dx.doi.org/10.1088/0953-8984/19/31/315220

[25] Wachter, P. (1994) Intermediate Valence and Heavy Fermions. In: Gschneidner Jr., K.A., Eyring, L., Lander, G.H. and Choppin, G.R., Eds., Handbook on the Physics and Chemistry of Rare Earths, Vol. 19, Lanthanides and Actinides: Physics II, Ch. 132, North-Holland Publishing, Amsterdam, 177-382.

[26] Junod, P., Menth, A. and Vogt, O. (1969) Revue des Propriété Magnétiques et Electroniques des Composés des Terres Rares avec les Anions du 5ieme Groupe du Système Périodique. Physik der kondensierten Materie, 8, 323-370. http://dx.doi.org/10.1007/bf02422864

[27] Wachter, P., Kaldis, E. and Hauger, R. (1978) Magnetic Exchange Interaction in the System GdP-GdS, Physical Review Letters, 40, 1404-1407. http://dx.doi.org/10.1103/PhysRevLett.40.1404

[28] Nagaev, E.L. (1967) Ground State and Anomalous Magnetic Moment of Conduction Electrons in an Antiferromagnetic Semiconductor. JETP Letters, 6, 18-20.

[29] Streit, P. and Wachter, P. (1970) Photoluminescence of EuSe and the Magnetic Polaron Model. Physik der kondensierten Materie, 11, 231-242.

[30] Trodahl, H.J., Preston, A.R.H., Zhong, J., Ruck, B.J., Strickland, N.M., Mitra, C. and Lambrecht, W.R.L. (2007) Ferromagnetic Redshift of the Optical Gap in GdN. Physical Review B, 76, 085211. http://dx.doi.org/10.1103/PhysRevB.76.085211

[31] Li, D.X., Haga, Y., Shida, H., Suzuki, T. and Kwon, Y.S. (1996) Electronic Transport Properties of Semimetallic GdX Single Crystals (X = P, As, Sb and Bi). Physical Review B, 54, 10483-10491. http://dx.doi.org/10.1103/PhysRevB.54.10483

[32] Monnier, R., Rhyner, J., Rice, T.M. and Koelling, D.D. (1985) Electron-Hole Liquid as a True Ground State. Physical Review B, 31, 5554-5556. http://dx.doi.org/10.1103/PhysRevB.31.5554

[33] Travaglini, G., Marabelli, F., Monnier, R., Kaldis, E. and Wachter, P. (1986) Electronic Structure of ScN. Physical Review B, 34, 3876-3882. http://dx.doi.org/10.1103/PhysRevB.34.3876

[34] Wachter, P., Unpublished Results.

[35] Duan, C.-G., Sabiryanov, R.F., Liu, L.J., Mei, W.N., Dowben, P.A. and Hardy, J.R. (2005) Strain Induced Half-Metal to Semiconductor Transition in GdN. Physical Review Letters, 94, 237201. http://dx.doi.org/10.1103/PhysRevLett.94.237201 